Journal for ImmunoTherapy of Cancer

\title{
Multicenter, double-blind, placebo- controlled trial of seviprotimut-L polyvalent melanoma vaccine in patients with post-resection melanoma at high risk of recurrence
}

Craig L Slingluff (D) , ${ }^{1}$ Karl D Lewis, ${ }^{2}$ Robert Andtbacka, ${ }^{3}$ John Hyngstrom, ${ }^{3}$ Mohammed Milhem, ${ }^{4}$ Svetomir N Markovic, ${ }^{5}$ Tawnya Bowles, ${ }^{6}$ Omid Hamid, ${ }^{7}$ Leonel Hernandez-Aya, ${ }^{8}$ Joel Claveau, ${ }^{9}$ Sekwon Jang, ${ }^{10}$ Prejesh Philips, ${ }^{11}$ Shernan G Holtan, ${ }^{12}$ Montaser F Shaheen, ${ }^{13}$ Brendan Curti (D) ${ }^{14}$ William Schmidt, ${ }^{15}$ Marcus O Butler, ${ }^{16}$ Juan Paramo, ${ }^{17}$ Jose Lutzky (D),${ }^{18}$ Arvinda Padmanabhan, ${ }^{19}$ Sajeve Thomas, ${ }^{20}$ Daniel Milton, ${ }^{21}$ Andrew Pecora, ${ }^{22}$ Takami Sato, ${ }^{23}$ Eddy Hsueh, ${ }^{24}$ Suprith Badarinath, ${ }^{25}$ John Keech, ${ }^{26}$ Sujith Kalmadi, ${ }^{27}$ Pallavi Kumar, ${ }^{28}$ Robert Weber, ${ }^{29}$ Edward Levine, ${ }^{30}$ Adam Berger, ${ }^{31}$ Anna Bar, ${ }^{32}$ J Thaddeus Beck, ${ }^{33}$ Jeffrey B Travers, ${ }^{34}$ Catalin Mihalcioiu, ${ }^{35}$ Brian Gastman, ${ }^{36}$ Peter Beitsch, ${ }^{37}$ Suthee Rapisuwon (D) , 38,39 John Glaspy, ${ }^{40}$ Edward C McCarron, ${ }^{41}$ Vinay Gupta, ${ }^{41}$ Deepti Behl, ${ }^{42}$ Brent Blumenstein, ${ }^{43}$ Joanna J Peterkin ${ }^{44}$

To cite: Slingluff CL, Lewis KD, Andtbacka R, et al. Multicenter, double-blind, placebo-controlled trial of seviprotimut-L polyvalent melanoma vaccine in patients with post-resection melanoma at high risk of recurrence. Journal for ImmunoTherapy of Cancer 2021;9:e003272. doi:10.1136/jitc-2021-003272

- Additional supplemental material is published online only. To view, please visit the journal online (http://dx.doi.org/10. 1136/jitc-2021-003272).

Accepted 06 September 2021

Check for updates

(c) Author(s) (or their employer(s)) 2021. Re-use permitted under CC BY-NC. No commercial re-use. See rights and permissions. Published by BMJ.

For numbered affiliations see end of article.

Correspondence to Dr Craig L Slingluff; cls8h@virginia.edu

\section{ABSTRACT}

Background Most patients with advanced melanomas relapse after checkpoint blockade therapy. Thus, immunotherapies are needed that can be applied safely early, in the adjuvant setting. Seviprotimut- $L$ is a vaccine containing human melanoma antigens, plus alum. To assess the efficacy of seviprotimut-L, the Melanoma Antigen Vaccine Immunotherapy Study (MAVIS) was initiated as a three-part multicenter, double-blind, placebocontrolled phase III trial. Results from part B1 are reported here.

Methods Patients with AJCC V.7 stage IIB-III cutaneous melanoma after resection were randomized 2:1, with stage stratification (IIB/C, IIIA, IIIB/C), to seviprotimut-L $40 \mathrm{mcg}$ or placebo. Recurrence-free survival (RFS) was the primary endpoint. For an hypothesized HR of 0.625 , one-sided alpha of 0.10 , and power $80 \%$, target enrollment was 325 patients.

Results For randomized patients ( $n=347$ ), arms were well-balanced, and treatment-emergent adverse events were similar for seviprotimut- $L$ and placebo. For the primary intent-to-treat endpoint of RFS, the estimated HR was 0.881 ( $95 \% \mathrm{Cl}: 0.629$ to 1.233 ), with stratified logrank $p=0.46$. However, estimated HRs were not uniform over the stage randomized strata, with HRs $(95 \%$ Cls) for stages IIB/IIC, IIIA, IIIB/IIIC of $0.67(95 \% \mathrm{Cl}: 0.37$ to 1.19$), 0.72$ (95\% Cl: 0.35 to 1.50$)$, and $1.19(95 \% \mathrm{Cl}$ : 0.72 to 1.97$)$, respectively. In the stage IIB/IIC stratum, the effect on RFS was greatest for patients $<60$ years old ( $\mathrm{HR}=0.324$ (95\% Cl: 0.121 to 0.864$))$ and those with ulcerated primary melanomas ( $\mathrm{HR}=0.493(95 \% \mathrm{Cl}: 0.255$ to 0.952$)$ ).
Conclusions Seviprotimut-L is very well tolerated. Exploratory efficacy model estimation supports further study in stage IIB/IIC patients, especially younger patients and those with ulcerated melanomas.

Trial registration number NCT01546571.

\section{INTRODUCTION}

Patients with resected stage IIB, IIC, or III melanoma are at high risk for recurrence and death after surgery. The only adjuvant therapy approved for stage IIB-IIC melanoma is high dose interferon, which prolongs recurrencefree survival (RFS) but has little or no proven impact on overall survival. ${ }^{1}$ Current National Comprehensive Cancer Network (NCCN) guidelines do not recommend interferon therapy for stage IIB-IIC melanoma. ${ }^{2}$ For stage III melanoma, adjuvant therapies approved by the United States Food and Drug Administration (U.S. FDA) include blockade of Programmed cell Death protein 1 (PD-1) with nivolumab or pembrolizumab, blockade of cytotoxic T-lymphocyte-Associated Protein 4 (CTLA-4) with ipilimumab, and inhibition of v-raf murine sarcoma viral oncogene homolog B1 (BRAF) and mitogen-activated protein kinase kinase (MEK) with dabrafenib and trametinib, respectively. However, for those with BRAF-wild type melanomas and 
low volume lymph node metastases, the risk: benefit ratio may not favor treatment with checkpoint blockade therapy. In addition, a significant portion of patients still recur despite approved adjuvant therapies. Thus, there is a need for effective systemic adjuvant therapy for patients with stage IIB-IIC melanoma and for subsets of patients with stage III melanoma.

Cancer vaccines offer promise to prevent melanoma recurrence by enhancing melanoma-specific immune responses. However, no cancer vaccine has yet proven effective for prolonging RFS or survival after melanoma resection. Seviprotimut-L (formerly POL-103A) was developed based on data from a prototype polyvalent melanoma vaccine comprised of shed antigens from four melanoma cell lines, administered with alum or interleukin (IL)-2 liposomes. That prototype vaccine contained multiple shared melanoma antigens, including MAGE-A3, MelanA, gp100, tyrosinase, melanocortin 1 receptor and tyrosinase-related protein $2^{34}$ and it induced antibody responses that were associated with improved clinical outcome. ${ }^{5}$ It also induced CD8 T cell responses to peptides restricted by Class I Major Histocompatability Complex (MHC) molecules. ${ }^{36}$ Over 600 patients were enrolled on trials testing versions of the prototype vaccine, with no safety concerns. In a small double-blind, placebo-controlled trial of a version of the prototype vaccine in patients with resected high-risk stage III melanoma $(n=38)$, RFS of the melanoma vaccinetreated patients was over twice as long as that of placebo vaccine-treated patients $(\mathrm{p}=0.03){ }^{7}$

The polyvalent vaccine has been reformulated as seviprotimut-L, prepared from antigens shed by three human melanoma cell lines administered with alum. A three-part, Phase III clinical trial program, termed Melanoma Antigen Vaccine Immunotherapy Study (MAVIS) was initiated. The trial program was originally designed to include two separate clinical trials: Part A to select between two dose levels of seviprotimut-L based on safety and immunogenicity, and Part B to evaluate safety and efficacy of the selected dose. A dose of $40 \mathrm{mcg}$ was selected in Part A based on safety and induction of serum antibody to melanoma antigens. In the course of conducting Part B (in 2016), the sponsor decided to split Part B into two parts-Part B1 to identify a signal of clinical efficacy, prior to initiating Part B2 which would be the definitive evaluation of clinical efficacy. Thus, Part B1 of MAVIS was a multicenter, double-blind, placebo-controlled trial to assess the efficacy of seviprotimut-L at that dose, with the primary endpoint of RFS in patients at high risk of recurrence after definitive surgical resection. The present manuscript reports the final clinical outcome data from Part B1 of the MAVIS trial.

\section{METHODS}

Part B1 of the MAVIS trial was a prospective doubleblind, randomized, placebo-controlled phase III clinical trial. The investigators and subjects were blinded as to treatment assignment; the placebo injections appeared identical to the seviprotimut-L injections.

\section{Participant eligibility}

Participants $18-80$ years of age were eligible if they had histologically confirmed AJCC Stage IIB, IIC, or III cutaneous melanoma (AJCC V.7) rendered clinically free of disease by surgery within 90 days. Patients with positive sentinel nodes were required to have a complete lymphadenectomy, except for patients on the Multicenter Selective Lymphadenectomy Trial II (MSLT-II, NCT00297895) ${ }^{8}$ who were randomized in that study to the observation arm. Also required were: Eastern Cooperative Oncology Group (ECOG) performance status 0-1, ability and willingness to give informed consent, normal organ function, and absence of major autoimmune disorders. Participants were required either to have known BRAF V600 mutation status or archival tissue available to test for BRAF mutation status. Participants were excluded for pregnancy, prior melanoma treatment other than surgery, regional radiation therapy or adjuvant interferon alfa-2b within 1 week of starting treatment, concurrent cancer therapy, concurrent immune therapy, chronic use of systemic corticosteroids, prior splenectomy, known HIV positivity, allergy to alum, or autoimmune disorders requiring therapy or with visceral involvement, or another malignancy within the prior 5 years (except adequately treated in situ squamous cell carcinoma, basal cell carcinoma, stage I or IIA melanoma, or carcinoma in situ of the cervix).

\section{Vaccine components and treatment regimen}

Seviprotimut-L is a polyvalent suspension melanoma cancer vaccine that includes multiple melanoma antigens shed from three human melanoma cell lines as the active pharmaceutical ingredient (API). These three lines are SFHM2, SFHM4, and SFHM8 and have previously been referred to as M20, M14, and SK-Mel-28, respectively: M20 was established from a metastatic melanoma ${ }^{9}$; M14 was established from an amelanotic metastatic lesion of a 33-year-old man and is BRAF-mutant $(\mathrm{V} 600 \mathrm{E})^{10}$; and SK-Mel-28 was originally established from an axillary lymph node of a 51-year-old man and is BRAF-mutant (V600E), with wild-type Neuroblastoma RAS viral oncolog gene homolog (NRAS). ${ }^{11-13}$ Additional information about each has been reported previously. ${ }^{14}$ For vaccine production, the material shed into serum-free culture medium by the cells was collected, pooled, concentrated, treated with non-ionic detergent Nonidet P-40 to disaggregate antigens, and ultracentrifuged to remove particulate matter. The supernatant was filter-sterilized, adjusted to the appropriate protein concentration, and bound to alum as an adjuvant. Seviprotimut-L is thus partially purified, being made of soluble shed antigens, to exclude the bulk of unrelated cellular material that is present in the cell cytoplasm or nucleus. The Drug Product was a nominal $0.8 \mathrm{~mL}$ suspension containing $0.05 \mathrm{mg} / \mathrm{mL}$ API with $1 \mathrm{mg} / \mathrm{mL}$ aluminum in aluminum hydroxide suspensions in $4 \mathrm{mM}$ phosphate-buffered saline and $0.9 \%$ 
sodium chloride, at $\mathrm{pH}$ 6.8. The Drug Product was packaged in Type I glass vials with Teflon-coated bromobutyl stoppers and was stored at $2^{\circ} \mathrm{C}-8^{\circ} \mathrm{C}$ (refrigerated) until use. The vaccine was used as is and required no reconstitution after warming to room temperature. The doses tested in Part A had included $40 \mathrm{mcg}$ and $100 \mathrm{mcg}$, where $40 \mathrm{mcg}$ protein had been the dose in the small randomied trial of a similar vaccine preparation. ${ }^{7}$

\section{Study design, power analysis, and objectives}

Part B1 of MAVIS was a two-arm, multicenter, randomized, double-blind, placebo-controlled trial with a goal of randomizing at least 325 patients in a 2:1 ratio for probability of assignment to the experimental arm. The primary endpoint was RFS. Secondary objectives included: (a) to assess the efficacy of treatment with seviprotimut-L compared with placebo with respect to overall survival $(\mathrm{OS})$; and (b) to verify the safety and tolerability of seviprotimut-L at the dose selected for Part B. As Part B1 was intended to identify a signal of clinical efficacy prior to embarking on the definitive Part B2, the $p$ value was set at 0.2, and hence fewer events/patients were required. For assessment of RFS, the study was powered with hypothesized HR of 0.625 , one-sided alpha of 0.10 , and power $80 \%$, with target assessment after 126 RFS events, based on the possibility of a delayed effect due to immunotherapy. ${ }^{15}$

Eligible patients received either seviprotimut-L at the dose determined from Part A $(40 \mu \mathrm{g})$ or placebo. Patients were stratified at randomization according to their stage of disease at enrollment (IIB/IIC vs IIIA vs IIIB/IIIC) to increase the likelihood of intended distribution between the study arms. Patients were treated every 2 weeks $\times 4$, then monthly $\times 4$, then every 3 months through month 24 (ie, weeks $0,2,4,6,8$, then months $3,4,5,6,9,12,15,18,21$, 24). Each subject was treated unless one of the following occurred: development of recurrent disease that did not meet the criteria for continued dosing, death, subject withdrawal, or study termination, whichever came first. Patients were followed for recurrence and survival. They were not routinely unblinded following recurrence or the end of treatment except to allow the subject to pursue other treatment options.

\section{Toxicity and dose-limiting toxicities}

Safety was assessed throughout the study. Adverse events (AEs) were reported from randomization through 30 days after the last study drug administration and were graded according to the National Cancer Institute Common Terminology Criteria for Adverse Events V.4. Relatedness of AEs to study drug was reported using WHO definitions: Probable, Possible, and Unlikely.

An adverse event or suspected adverse reaction was considered 'serious' (SAE) if, in the view of either the investigator or sponsor, it resulted in any of the following outcomes: death, a life-threatening adverse event, inpatient hospitalization or prolongation of existing hospitalization, a persistent or significant incapacity or substantial disruption of the ability to conduct normal life functions, or a congenital anomaly/birth defect. Other events would be considered serious when, based on appropriate medical judgment, they may jeopardize the patient or subject and may require medical or surgical intervention to prevent one of the outcomes listed in this definition.

\section{Clinical outcome}

The date of recurrence was defined as the earliest date a recurrence was suspected, with biopsy confirmation, except in rare cases where biopsies were not clinically appropriate (eg, brain metastases). Recurrence events included appearance of locoregional metastasis, distant metastasis, or a new primary melanoma. Since the validation of recurrence primarily relied on biopsies, there was no central reading of CT scans to confirm recurrence.

Recurrence assessments were to be based on crosssectional imaging every 6 months. Serum lactate dehydrogenase (LDH) was also monitored every 3 months, and LDH elevations $\geq 1.5 \times$ upper limit of normal were followed up with radiological scans, provided there were no other obvious medical reasons for the elevated LDH.

\section{Statistical methods}

The primary analysis outcome is RFS time, computed as the number of days from the date of randomization to the earliest of recurrence or death. Patients without recurrence or death were censored at the date last assessed for recurrence. All analyses are based on the intent-to-treat (ITT) principle. The primary analysis is the stratified logrank test, with the stage at randomization as stratifier. HR estimates and their 95\% CIs are from proportional hazard regression models using stage at randomization as stratifier as applicable. Sensitivity and exploratory analyses included effect modification assessment for the existence of heterogeneity of RFS effect estimated across subsets defined by baseline attributes and is based on a proportional hazard regression with terms for baseline attribute, arm, and one or more terms as necessary for the interaction between baseline attribute and arm. In effect modification assessments, an interaction $p$ value in the neighborhood of 0.1 or less is regarded as evidence of effect modification (this interaction $p$ value is to be interpreted as a measure of the contribution of interaction to the fit of the model). Graphs show the Kaplan-Meier estimators of the time-to-event distributions. Beyond the primary analysis, all analyses are presented as exploratory with arm effect estimates and 95\% CIs.

\section{RESULTS \\ Patients}

Three hundred and forty-seven patients at 65 centers in the USA and Canada were randomized January 2015 through August 2016. Arms were well-balanced (table 1). About one-third of patients had a BRAF mutation. Only $5 \%$ had had any prior melanoma therapy other than surgery, and these were primarily radiotherapy. None had 
Table 1 Enrollment, demographics, and clinical features

\begin{tabular}{|c|c|c|}
\hline & Seviprotimut-L & Placebo \\
\hline $\mathrm{N}$ & 230 & 117 \\
\hline Age: median (range) & $58(18,80)$ & $56(26,80)$ \\
\hline Sex: \% female & 42 & 44 \\
\hline Race: \% white & 99 & 100 \\
\hline \multicolumn{3}{|l|}{ Prior melanoma therapy (\%) } \\
\hline Any & 5.2 & 4.3 \\
\hline Radiotherapy & 4.3 & 4.3 \\
\hline \multicolumn{3}{|l|}{ Tumor location (\%) } \\
\hline Extremities & 35 & 37 \\
\hline Head and neck & 26 & 21 \\
\hline Trunk & 37 & 41 \\
\hline Other & 2.6 & 0.9 \\
\hline Ethnicity: \% Hispanic or Latino & 3 & 3.4 \\
\hline ECOG performance status 0 (\%) & 86 & 86 \\
\hline Completed lymphadenectomy (\%) & 64 & 64 \\
\hline Median Breslow depth (mm) & 2.8 & 3 \\
\hline \multicolumn{3}{|l|}{ T staging (\%) } \\
\hline T0 or TX & 4.8 & 5.1 \\
\hline T1-T2a & 29 & 27 \\
\hline $\mathrm{T} 2 \mathrm{~b}-\mathrm{T} 4 \mathrm{a}$ & 48 & 55 \\
\hline $\mathrm{T} 4 \mathrm{~b}$ & 18 & 17 \\
\hline \multicolumn{3}{|l|}{$\mathrm{N}$ staging (\%) } \\
\hline NO & 32 & 32 \\
\hline $\mathrm{N} 1 \mathrm{a}$ & 36 & 30 \\
\hline $\mathrm{N} 1 \mathrm{~b}-\mathrm{N} 2 \mathrm{~b}$ & 19 & 23 \\
\hline N2c/N3 & 13 & 15 \\
\hline \multicolumn{3}{|l|}{ Summary AJCC stage (V.7, \%) } \\
\hline IIB/IIC & 32 & 32 \\
\hline IIIA & 30 & 29 \\
\hline IIIB/IIIC & 38 & 39 \\
\hline BRAF mutant* (\%) & 35 & 33 \\
\hline
\end{tabular}

*BRAF mutation status known for $93 \%, 96 \%$, and $94 \%$ of patients receiving vaccine, placebo, or total. Values shown above are percentages of all.

prior immunotherapy, small molecule targeted agents, antibody therapy, or anti-angiogenic therapy. Patients on the MSLT-II trial were allowed to enroll only after amendment 3. Only one, on the observation arm, is documented to be enrolled also on MSLT-II. The data in this manuscript were collected after datalock in January 2020, and findings were provided to the coauthors in April 2020, after which analyses and statistical review led to the present manuscript.

\section{Adverse events and safety assessment}

Over $95 \%$ of patients on both study arms had one or more treatment-emergent AE's, and $71 \%$ had one or more treatment-related AEs (TRAEs), all of which are detailed in online supplemental table 1 . The vast majority were grade 1 , with fewer grade 2 TRAEs and very few grade 3 TRAEs. The TRAEs observed in $3 \%$ or more of patients overall, listed in table 2, included local symptoms at the injection sites (erythema, pruritus, pain, swelling, bruising, induration, and rash) and systemic symptoms (fatigue, headache, nausea, pruritus, myalgia, rash, influenza-like illness symptoms, and diarrhea). The incidence of those AEs and associated AE categories were similar between the two study arms. The only AE with slightly higher incidence in the vaccine arm was injection site pruritus (20\% vs $12 \%$ ). There were no grade 4-5 TRAEs and no treatment-related SAEs (table 3). Only one patient discontinued therapy for a TRAE: a macular rash on the vaccine arm. Sixty-two per cent completed all 24 months of therapy; 32\% discontinued early for disease progression (table 3 ). Evidence of autoimmune disease in the eyes and skin was assessed every 6 months. Evidence of ocular autoimmune disease was reported only in one participant at month 12 on the vaccine arm and in none on the placebo arm. Evidence of autoimmune disease in the skin was observed in small numbers on both vaccine and placebo arms, with no evidence of a treatment-related effect (online supplemental table 2).

\section{RFS}

The primary analysis in this report is based on data with 149 RFS events at the data cut-off date. For this primary analysis, the estimated HR is 0.881 (95\% CI: 0.629 to 1.233 ), with $\mathrm{p}=0.460$. Kaplan-Meier estimates are shown in figure 1A. Thus, the trial failed to meet the planned primary statistical criterion. However, there was notable quantitative heterogeneity in HR estimates across stagespecific randomization strata. For stages IIB/IIC $(n=111)$, IIIA ( $\mathrm{n}=103)$, IIIB/IIIC $(\mathrm{n}=133)$, respectively, these HR estimates (and 95\% CIs) are 0.666 (95\% CI: 0.373 to 1.189 ), 0.721 (95\% CI: 0.347 to 1.496 ) , and 1.191 (95\% CI: 0.720 to 1.970$)$. Figure 1B shows the Kaplan-Meier estimates by stage and arm. The sites of metastasis were defined as locoregional or distant for 137 patients, including 47, 24, and 66 for stages IIB/IIC, IIIA, IIIB/C, respectively: of these, relapses were distant in $26(55 \%), 10(42 \%)$, and $37(56 \%)$, respectively (data not shown). Patients were stratified and randomized for enrollment on this trial based on AJCC V.7 staging. AJCC V.8 classifies stage IIB and IIC patients the same as V.7; so, analyses of this subset are the same using AJCC V.8. There were changes for AJCC V.8 in substaging stage III patients, but reanalysis of outcomes in stage III patients is inconsistent with the randomization stratification. The finding of a potentially clinically meaningful stage IIB/IIC point estimate and the consistent and distinct separation between arms for stage IIB/IIC as seen in the Kaplan-Meier estimates (figure 1B) led to further exploration of the stage IIB/ IIC patients with the intent to inform planning the Part B2 phase III trial. Additional factors explored as potential effect modifiers within the stage IIB/IIC group included age, sex, tumor location, thickness, ulceration, BRAF mutation status, and LDH. Suggestion of the existence of 


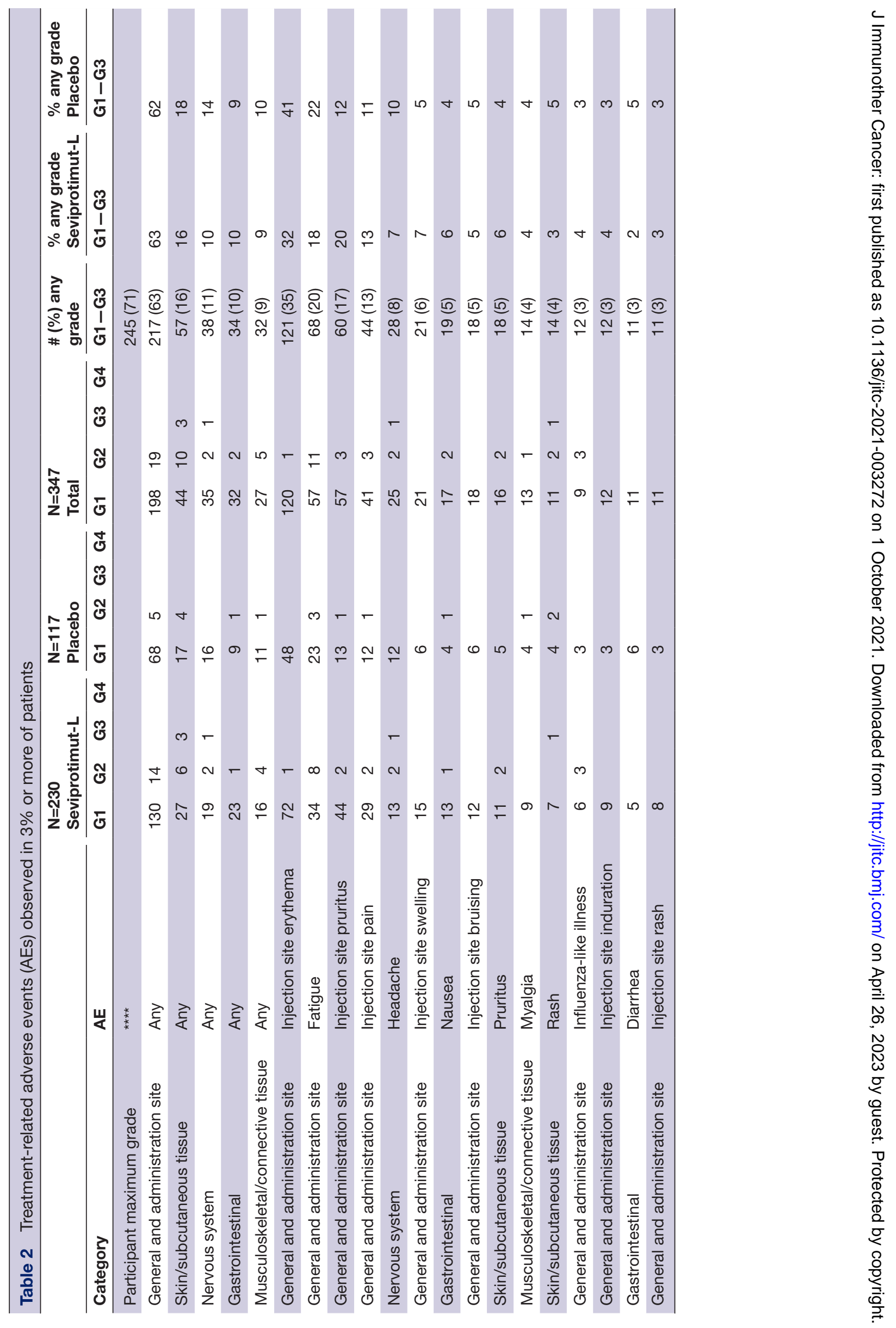


Table 3 Summary of adverse events (AEs) and protocol discontinuation

\begin{tabular}{|c|c|c|c|}
\hline & Seviprotimut-L & Placebo & Total \\
\hline $\mathrm{N}$ & 230 & 117 & 347 \\
\hline AEs & $96 \%$ & $97 \%$ & $96 \%$ \\
\hline Grade 3 AEs & $12 \%$ & $9 \%$ & $11 \%$ \\
\hline Grade 4-5 AEs & $0 \%$ & $0 \%$ & $0 \%$ \\
\hline $\begin{array}{l}\text { Treatment-related AEs } \\
\text { (TRAEs) }\end{array}$ & $70 \%$ & $73 \%$ & $71 \%$ \\
\hline $\begin{array}{l}\text { Treatment-related serious } \\
\text { AEs }\end{array}$ & $0 \%$ & $0 \%$ & $0 \%$ \\
\hline $\begin{array}{l}\text { AEs leading to d/c study } \\
\text { drug }^{*}\end{array}$ & $0.9 \%$ & $0.9 \%$ & $0.9 \%$ \\
\hline $\begin{array}{l}\text { TRAEs leading to } d / c \text { study } \\
\text { drug }\end{array}$ & $0.4 \%$ & $0 \%$ & $0.3 \%$ \\
\hline $\begin{array}{l}\text { Completed } 24 \text { months of } \\
\text { treatment }\end{array}$ & $61.3 \%$ & $63.2 \%$ & $62.0 \%$ \\
\hline $\begin{array}{l}\text { D/C early for progressive } \\
\text { disease }\end{array}$ & $31.7 \%$ & $31.6 \%$ & $31.7 \%$ \\
\hline $\begin{array}{l}\text { D/C early for withdrawal of } \\
\text { consent }\end{array}$ & $3.9 \%$ & $1.7 \%$ & $3.2 \%$ \\
\hline D/C early for TRAE & $0.4 \%$ & $0 \%$ & $0.3 \%$ \\
\hline $\begin{array}{l}\text { Lost to follow-up, } \\
\text { pregnancy, other cancer }\end{array}$ & $2.6 \%$ & $3.4 \%$ & $2.9 \%$ \\
\hline Total & $100 \%$ & $100 \%$ & $100 \%$ \\
\hline
\end{tabular}

${ }^{*} A E s$ leading to discontinuation $(d / c)$ of study drug included one TRAE (macular rash) on the vaccine arm, and development of adenocarcinoma of the colon on the vaccine arm and squamous cell carcinoma of the larynx on the placebo arm.

an effect modification was found for age as $<60$ versus $\geq 60$ years and for the presence of ulceration, with interaction $p$ values of 0.0581 and 0.1136 , respectively. The findings are illustrated for age in figure 1C,D for the ITT data set and stage IIB/C group, respectively.

Findings regarding effect modification by the presence of ulceration and age for the stage IIB/C stratum are illustrated in figure 2. For this stratum, the HR for ulcerated melanomas was 0.493 (95\% CI: 0.255 to 0.952$)$, as shown in figure 2A. The interactions between age $(\leq 60$ and $>60$ years) and ulceration for the stage IIB/IIC stratum are further detailed in figure 2B,C. For those under age 60 with ulcerated melanomas, specifically, the HR was 0.213 (95\% CI: 0.065 to 0.702 ) favoring the vaccine arm.

\section{Overall survival}

OS was a secondary endpoint. With 41 deaths, there is reduced sensitivity for detecting treatment effects on OS; however, by ITT analysis, the estimated HR favors the vaccine arm ( $\mathrm{HR}=0.64$ (95\% CI: 0.34 to 1.18$)$, figure $3 \mathrm{~A})$. Also, the ranking across disease stages for OS is similar to the quantitative relationships in the RFS analyses. Analysis of subgroups based on planned stratification by stage (figure 3B) replicated the RFS trend, with evidence of a strong HR effect in Stage IIB/IIC patients $(n=111$, HR 0.338 (95\% CI: 0.117 to 0.975 ), figure 3B,D). Also, the survival HR for the 191 patients $<60$ years old was 0.412 (95\% CI: 0.167 to 1.014 , figure 3C,D), while for the 156 patients $\geq 60$ years old, the HR was 0.91 (95\% CI: 0.38 to 2.17, figure $3 \mathrm{C}, \mathrm{D}$ ). For stage IIB/IIC stratum, the survival HR for the 52 patients $<60$ years old was 0.216 (95\% CI: 0.039 to 1.19 ), and for the 59 patients $\geq 60$ years old, the HR was 0.49 (95\% CI: 0.12 to 1.98 , figure 3E). The survival HR for 160 patients with ulcerated primary melanomas was 0.51 (95\% CI: 0.22 to 1.16 ), and for the stage IIB/ IIC stratum, the survival HR for 84 patients with ulcerated melanomas was 0.345 (95\% CI: 0.10 to 1.14 ; not shown).

\section{DISCUSSION}

Seviprotimut-L treatment was very well tolerated, with no SAEs and without a clear signal for increases in TRAEs compared with placebo injections. Part B1 of the MAVIS trial was designed to obtain preliminary data on clinical impact of seviprotimut-L, at $40 \mu \mathrm{g} / \mathrm{dose}$, as measured by RFS. The study was powered to detect a HR of 0.625 , with one-sided alpha of 0.10 , and power $80 \%$. The target of 126 RFS events was met. The HR for RFS for the ITT data set was 0.88 (95\% CI: 0.63 to 1.23); so, these data do not support benefit of seviprotimut-L for the whole patient data set. However, a planned randomization stratification by stage identified a subset, representing $32 \%$ of the study data set (stage IIB/IIC), for whom there was a trend toward longer RFS with vaccine. Further, subgroup analyses by age and ulceration suggest benefit for patients under age 60 and, for the stage IIB/C stratum, those with ulcerated primary melanomas. Analysis of impact on OS was limited by the smaller number of events, but the HR of 0.64 is promising. For the Stage IIB/IIC subset, OS was longer with seviprotimut-L, with HR 0.338 (95\% CI: 0.117 to 0.975 ). Also, OS trended longer for patients with stage IIB/IIC melanoma under age 60 (HR 0.216). In the recently reported S1404 study evaluating adjuvant pembrolizumab versus standard of care (ipilimumab or high-dose interferon) for patients with resected stage III-IV melanoma, significant improvement in RFS did not translate to a significant impact on OS. ${ }^{16}$ Thus, the promising early RFS and OS data for stage IIB/C patients in the MAVIS trial are particularly promising. These data support selection of stage IIB/C patients for the definitive final part B2 of the MAVIS phase III trial to test seviprotimut-L, with stratification by age and ulceration.

The seviprotimut-L vaccine contains soluble antigens shed from three human melanoma cell lines, in a preparation that is readily scaled up for large patient populations. The preparation contains multiple defined antigens shared among melanomas from many patients, including melanocytic differentiation proteins and cancer-testis antigens. ${ }^{36}$ There is evidence that such proteins can serve as relevant tumor-rejection antigens: vaccination with a single shared melanocytic antigen improved progressionfree survival of patients with melanoma when added to systemic interleukin-2 therapy in a randomized trial, ${ }^{17}$ and vaccination with a mixture of peptides from shared 

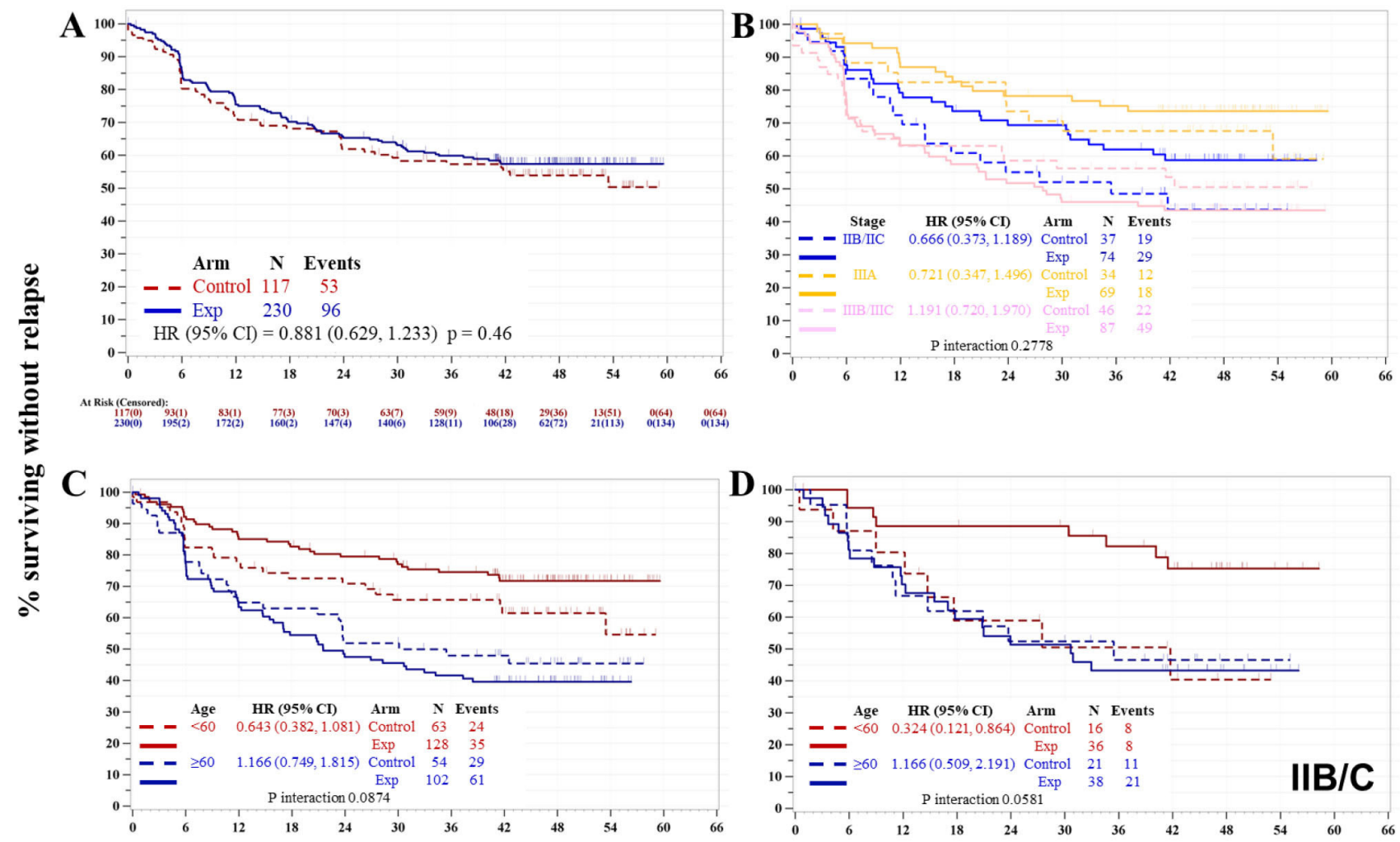

RFS time (months, at cutoff)

Figure 1 Recurrence-free survival (RFS) by arm, stage, and age. Kaplan-Meier estimates of RFS are plotted, with analysis results in the legend and risk set and censoring accumulation below the time axis. The designation '(cut-off)' indicates possible data truncation based on designated cut-off date. (A) RFS by arm, stratified by stage, for the intent-to-treat (ITT) data set, (B) RFS by arm and stage for the ITT data set, (C) RFS by arm and age for the ITT data set, (D) RFS by arm and age for Stage IIB/C stratum. $\mathrm{P}$ values by logrank test. $\mathrm{P}$ value, $\mathrm{HR}$ and $95 \% \mathrm{Cl}$ are based on a univariate Cox regression model assuming proportional hazards with treatment, age, and treatmentxage as factors, stratified for the randomisation stratification variable of disease stage.

melanocytic and cancer testis proteins has induced durable clinical benefit in patients with advanced melanoma. $^{18}$ Also, adoptive cell therapy targeting shared cancer-testis antigens has induced durable clinical benefit in melanoma and other cancers. ${ }^{19} 20$ A limitation of the approach is the complexity of the antigen composition, which limits the ability to test for immune responses that are most relevant for tumor control. However, an advantage of the seviprotimut-L approach is that it is not limited to a small number of peptides or proteins and does not require selection of patients based on human leukocyte antigen (HLA) expression.

The subgroup analyses in this report are obligatory and inform directions for future study of this vaccine. In stage IIB/IIC patients, there was a trend to prolonged RFS (HR 0.666, figure 1B) and prolonged OS (HR 0.338, figure $3 \mathrm{~B}$ ) for those who received seviprotimut-L. The reasons for these different results based on stage are not clear. It is true that in prior trials with PD-1 inhibitors, and $\mathrm{BRAF} / \mathrm{MEK}$ inhibition, there has been benefit across a range of stages, for stage III and IV, as evidenced by FDA approvals in these settings. Vaccines may have a greater chance of benefit in earlier stages. Patients without lymph node metastases may have more intact immune function.
It has been hypothesized that for melanoma to establish metastases in regional nodes, the primary tumor must first induce immune dysregulation in those nodes. ${ }^{21}$ Regardless, these findings support limiting the design of the future part B2 trial to patients with stage IIB/IIC melanoma, who are those for whom no systemic adjuvant therapy is currently recommended.

Other subset findings are suggestions of benefit with seviprotimut-L for patients younger than age 60 and for those with ulcerated primary melanomas. In particular, for stage IIB/IIC patients, RFS was more favorable with seviprotimut-L for age $<60$ (HR 0.324, figure 1D) and for ulcerated melanomas (HR 0.493, figure 2A). The rationale for improved outcomes in younger patients is supported by prior experience. Older individuals develop immune senescence that decreases immune responsiveness, ${ }^{22}$ and they have lower immune response rates to peptide vaccines for melanoma. ${ }^{23}$ Thus, the present study supports evaluating the clinical impact of seviprotimut-L in patients under age 60 in the subsequent MAVIS clinical trial. Interestingly, PD-1 blockade has been reported to be more effective in patients over age $60,{ }^{24}$ suggesting the possible benefit of combining vaccine and PD-1 blockade specifically in older patients. The improved outcome in 

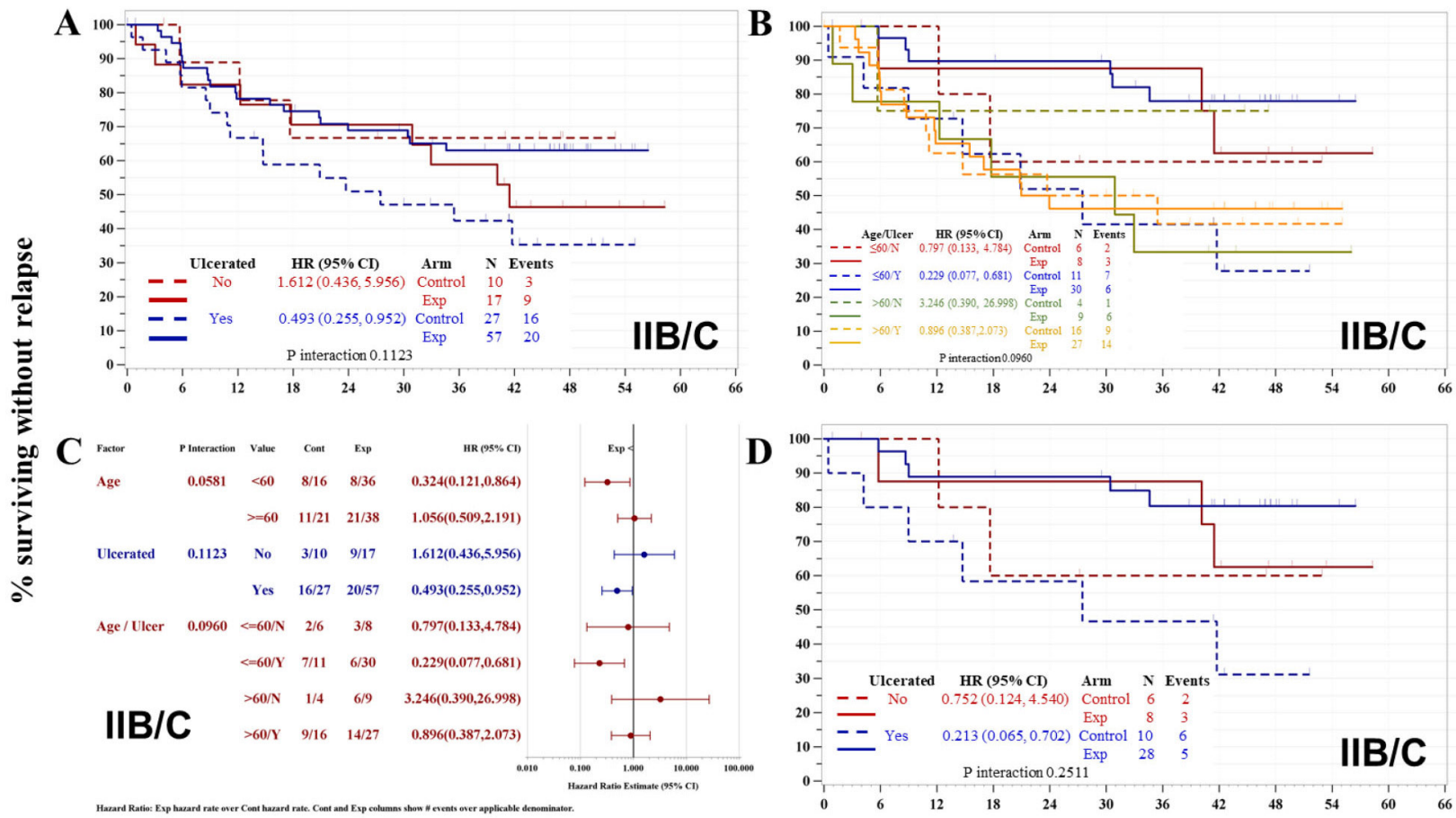

RFS time (months, at cutoff)

Figure 2 Recurrence-free survival (RFS) by arm, age, and ulceration for stage IIB/IIC stratum. Kaplan-Meier estimates of RFS are plotted for stage IIB/IIC patients (A) by arm and ulceration, and (B) by arm, age and ulceration. Shown in (C) is a forest plot for stage IIB/IIC patients as a function of age, ulceration, and both. In (D) is the Kaplan-Meier estimate for RFS for stage IIB/IIC patients under age 60 , by arm and ulceration. $P$ values by logrank test.

patients with ulcerated primary melanomas is consistent with prior reports of improved outcome with another cancer vaccine and with interferon therapy for patients with ulcerated melanomas. ${ }^{25} 26$ The finding in this trial is also promising and may guide future trial design as well.

Several prior randomized clinical trials of cancer vaccines have been disappointing, including the Canvaxin trials of an allogeneic whole melanoma cell vaccine administered with Bacillus Calmette-Guérin adjuvant. ${ }^{27} 28$ The definitive phase III trials of that vaccine were performed in patients with stage III and stage IV melanoma, which were negative. The lack of an efficacy signal for stage III patients in the present trial is consistent with that finding. Also, a randomized trial of an autologous heat-shock protein vaccine vitespen in patients with stage IV melanoma, also revealed no impact on overall survival. $^{29}$ Thus, the promise of cancer vaccines for melanoma remains to be realized. On the other hand, a vaccine approach has been approved in hormone-refractory prostate cancer, ${ }^{30}$ and a phase III trial of a peptide vaccine plus highdose IL-2 significantly prolonged progression-free survival of patients with advanced melanoma, compared with IL-2 alone. ${ }^{31}$ The data from Part B1 of the MAVIS trial do not meet the target impact on RFS for the whole ITT population, but promising trend to longer overall survival for the ITT population (HR 0.64) support continued investigation of this approach. A goal of the present study was to assess for evidence of clinical benefit to guide a definitive Part B2 randomized phase III trial. Failure of prior trials may also have related to the study population. The present study has identified subsets who for whom there is promising preliminary evidence of efficacy of this vaccine approach, which will thus guide the follow-up definitive trial.

Limitations of the present study include its modest sample size and the negative findings overall for impact on RFS. Also, the composition of the vaccine, being derived from cell-associated proteins, is not conducive to laboratory correlates to assess immune response to defined antigens. The present B1 trial did not include analyses of immune responses; so, there are no data on the strength of the immune responses or their association with clinical outcome. BRAF mutation status has been associated with clinical outcome and response to checkpoint blockade therapy, as well as BRAF/MEK inhibitor therapy. However, during this trial, it was not routine to assess BRAF mutation status for resected stage II-III melanomas; yet, the arms were well-matched for BRAF mutation status overall in those for whom it was known (table 1 ). There may be value in stratifying also for BRAF status in subsequent vaccine trials.

In summary, this final report of data from part B1 of the prospective randomized multicenter MAVIS clinical trial of seviprotimut-L provides guidance useful for design of phase III part B2 of the MAVIS clinical trial program. Subgroup efficacy analyses identified three groups who may benefit from seviprotimut-L: those with AJCC (V.7) stage IIB/C melanoma, those under age 60 , and those with ulcerated melanomas. These 

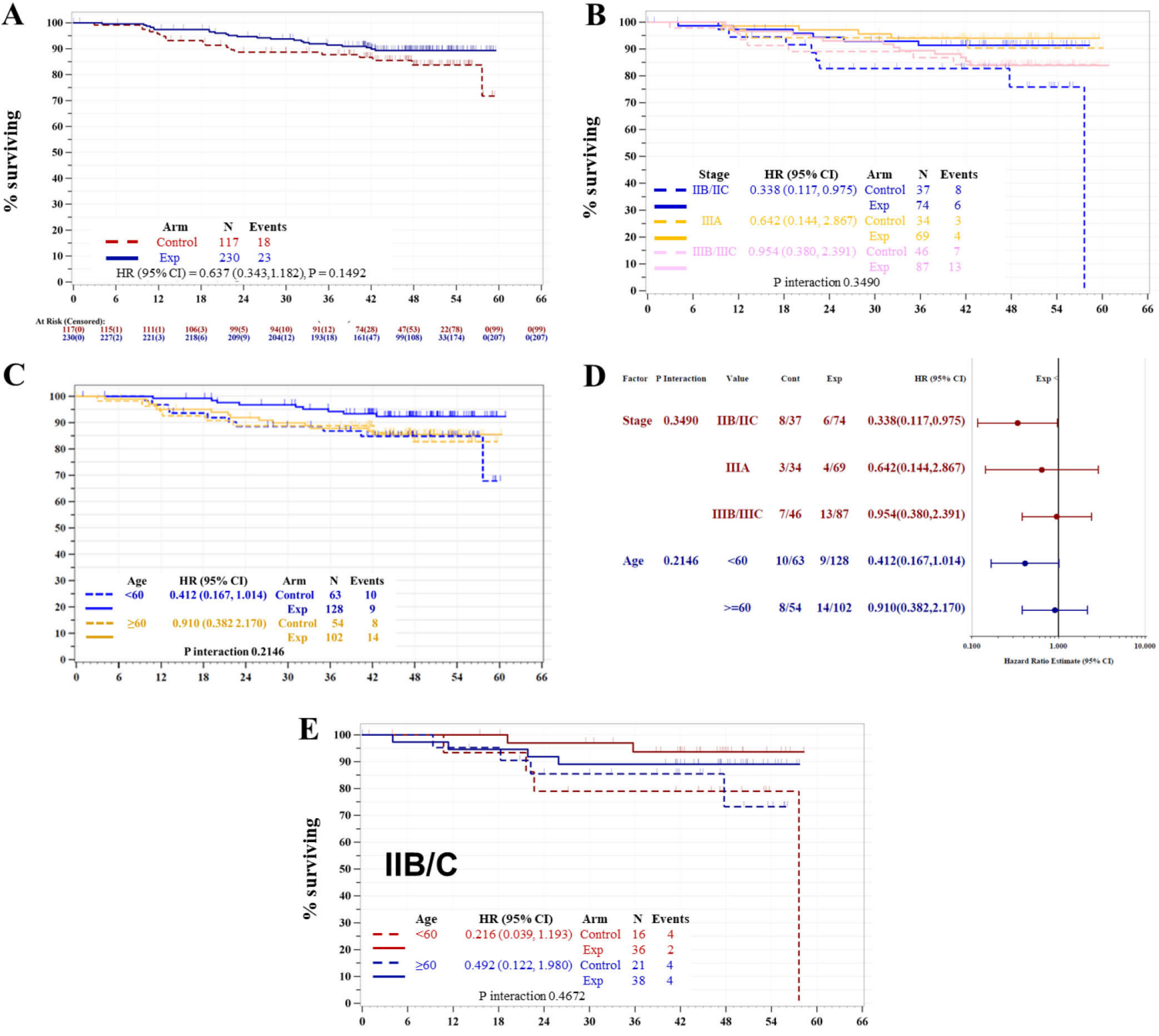

OS time (months, at cutoff)

Figure 3 Survival effect modification by arm, age, and stage. Kaplan-Meier estimates of overall survival (OS) are plotted (A) for all intent-to-treat (ITT) patients stratified by stage, by arm, (B) for all ITT patients by arm and stage, and (C) for all ITT patients by arm and age. Shown in (D) is a forest plot for all ITT patients by stage and by age. In (E) is the Kaplan-Meier estimate for OS for stage IIB/IIC patients by arm and age. $P$ values by logrank test.

data support proceeding to the definitive final part B2 of the MAVIS phase III trial to test seviprotimut-L for stage IIB/C patients, with stratification by age and ulceration. Currently available adjuvant therapies for patients with stage III melanoma include immune checkpoint blockade with antibodies to CTLA- 4 or PD-1, and small molecule inhibitors of V600-mutated BRAF and MEK. However, they are not currently approved for patients with resected stage IIB/C melanoma. Clinical trials currently underway evaluating PD-1 blockade for stage IIB/IIC melanoma include Keynote 716 (NCT03553836) and Checkmate 76K (NCT04099251). A recent press release of Keynote 716 reports that adjuvant pembrolizumab met its primary endpoint for RFS for patients with stage IIB/IIC melanoma ${ }^{32}$; so, the FDA is likely to review of those data in the near future. However, in patients without metastatic disease, the tolerance of SAEs is less than in patients with advanced melanoma. Though PD-1 blockade is well tolerated by most patients, severe and lifethreatening toxicities can occur, ${ }^{33}$ including cardiac and neuromuscular toxicities, which have a high mortality risk, ${ }^{34}$ as well as diabetes ${ }^{35}$ and other endocrinopathies. ${ }^{36}$ To our knowledge, there are no phase III BRAF/MEK inhibitor adjuvant trials in stage IIB/C melanoma. The toxicity of dabrafenib/trametinib in the COMBI-AD adjuvant melanoma trial in Stage III patients led to SAEs in $36 \%$ of patients and permanent discontinuation of study drug in $26 \%$ of patients. ${ }^{37}$ On the other hand, vaccination 
with seviprotimut-L has an advantage of very low toxicity without significant immune-related adverse events, and no significant increase in toxicity over placebo. Thus, if definitive evaluation of this vaccine therapy confirms clinical benefit in patients with stage IIB/C melanoma, the low toxicity of this approach will be a valuable option for these patients.

\section{STATEMENT OF TRANSLATIONAL RELEVANCE}

Seviprotimut-L, a vaccine containing antigens from human melanoma cells, is being evaluated for efficacy in this randomized, placebo-controlled trial for patients with AJCC v7 stage IIB-III cutaneous melanoma after resection. Treatment-emergent adverse events (AEs) were similar for seviprotimut-L and placebo patients. For the primary intent-to-treat (ITT) endpoint of RFS, the estimated HR was 0.881 (95\% CI: 0.629,1.233). However, subgroup efficacy analyses identified three groups who may benefit from Seviprotimut-L: those with AJCG stage IIB/IIC melanoma, those under age 60, and those with ulcerated melanomas. These data support proceeding to the definitive final part B2 of the MAVIS phase III trial to test seviprotimut-L for stage IIB/C patients, with stratification by age and ulceration. In summary, seviprotimut-L is very well tolerated. Exploratory efficacy model estimation supports further study in stage IIB/IIC patients, especially younger patients and those with ulcerated melanomas.

\section{Author affiliations}

${ }^{1}$ Department of Surgery, University of Virginia School of Medicine, Charlottesville, Virginia, USA

${ }^{2}$ University of Colorado - Anschutz Medical Campus, Aurora, Colorado, USA

${ }^{3}$ Huntsman Cancer Institute Cancer Hospital, Salt Lake City, Utah, USA

${ }^{4}$ The University of lowa Hospitals and Clinics, lowa City, lowa, USA

${ }^{5}$ Mayo Clinic Rochester, Rochester, Minnesota, USA

${ }^{6}$ Intermountain Medical Center, Murray, Utah, USA

${ }^{7}$ Cedars-Sinai Medical Center Angeles Clinic and Research Institute, Santa Monica, California, USA

${ }^{8}$ Department of Medicine, Washington University School of Medicine in Saint Louis, Saint Louis, Missouri, USA

${ }^{9} \mathrm{CHU}$ de Quebec-Universite Laval, Quebec, Québec, Canada

${ }^{10}$ Department of Medical Oncology, Inova Health System, Falls Church, Virginia, USA

${ }^{11}$ University of Louisville, Louisville, Kentucky, USA

${ }^{12}$ University of Minnesota Academic Health Center, Minneapolis, Minnesota, USA

${ }^{13}$ University of Arizona Medical Center - University Campus, Tucson, Arizona, USA

${ }^{14}$ Earle A Chiles Research Institute, Providence Portland Medical Center, Portland,

Oregon, USA

${ }^{15}$ Bend Memorial Clinic, Bend, Oregon, USA

${ }^{16}$ Princess Margaret Hospital Cancer Centre, Toronto, Ontario, Canada

${ }^{17}$ Mount Sinai Medical Center, Miami Beach, Florida, USA

${ }^{18}$ Department of Oncology, Sylvester Comprehensive Cancer Center, Miami, Florida, USA

${ }^{19}$ Baptist Health Lexington, Lexington, Kentucky, USA

${ }^{20}$ MD Anderson Cancer Center Orlando, Orlando, Florida, USA

${ }^{21}$ Investigative Clinical Research of Indiana, Indianapolis, Indiana, USA

${ }^{22}$ Department of Oncology, John Theurer Cancer Center, Hackensack, New Jersey, USA

${ }^{23}$ Department of Medical Oncology, Thomas Jefferson University, Philadelphia,

Pennsylvania, USA

${ }^{24}$ St. Louis University Hospital, St. Louis, Missouri, USA

${ }^{25}$ Cancer Specialists of North Florida, Jacksonville, Florida, USA

${ }^{26}$ Multicare Institute for Research and Innovation, Tacoma, Washington, USA

${ }^{27}$ Ironwood Cancer and Research Centers, Chandler, Arizona, USA
${ }^{28}$ Harry and Jeanette Weinberg Cancer Institute at Franklin Square, Baltimore, Maryland, USA

${ }^{29}$ St. Mary's Hospital and Medical Center, San Francisco, California, USA

${ }^{30}$ Wake Forest School of Medicine, Winston-Salem, North Carolina, USA

${ }^{31}$ Rutgers Cancer Institute of New Jersey, New Brunswick, New Jersey, USA

${ }^{32}$ Oregon Health \& Science University, Portland, Oregon, USA

${ }^{33}$ Department of Medical Oncology, Highlands Oncology Group, Fayetteville,

Arkansas, USA

${ }^{34}$ Premier Health Partners Inc, Dayton, Ohio, USA

${ }^{35}$ Royal Victoria Hospital, Montreal, Québec, Canada

${ }^{36}$ Department of Plastic Surgery, Cleveland Clinic, Cleveland, Ohio, USA

${ }^{37}$ Cancer Solutions, Dallas, Texas, USA

${ }^{38}$ Department of Oncology, Georgetown University Medical Center, Washington, District of Columbia, USA

${ }^{39}$ Department of Hematology/Oncology, MedStar Washington Hospital Center, Washington, District of Columbia, USA

${ }^{40}$ University of California Los Angeles, Los Angeles, California, USA

${ }^{41}$ MedStar Franklin Square Medical Center, Baltimore, Maryland, USA

${ }^{42}$ Sutter Institute for Medical Research, Sacramento, California, USA

${ }^{43}$ Trial Architecture Consulting, Chevy Chase, Maryland, USA

${ }^{44}$ Polynoma, San Diego, California, USA

Acknowledgements We acknowledge participation of all the patients and their families, support of all investigators and clinical coordinators responsible for enrolling patients to this trial, Jean-Claude Bystryn for his initial work on this vaccine strategy, and funding support from Polynoma. We also acknowledge the support of the Maryland Melanoma Center (Amy Avergas, MSIT, MBA; Ed McCarron, MD; Vinay Gupta, MD); Kathleen Haden, NP, at the University of Virginia Cancer Center.

Contributors All authors except JJP and BB enrolled patients, and collected data on adverse events and clinical outcomes at their institutions. CLS was overall principal investigator for the trial and reviewed final study data and worked with $\mathrm{BB}$ and the other authors to write the manuscript. BB was the study statistician. He provided statistical analysis and figures. JJP oversaw the study from the perspective of the sponsor. All authors have approved the manuscript.

Funding This trial was funded by the sponsor, Polynoma.

Competing interests CLS discloses research funding to his University from Merck, Celldex, and GlaxoSmithKline; research support in kind to his University from Theraclion, 3M, Merck, and Celldex; Scientific Advisory Board role with Immatics (prior), and Curevac; principal investigator role for Polynoma with compensation to his university, related to this manuscript; and patent royalties as co-inventor of peptides for use in cancer vaccines (patents held by the UVA Licensing and Ventures Group). KDL received research funding from Polynoma. JH served on an advisory board for Nektar and BMS in 2020. SNM has received research grants from BMS and from Sorrento Pharma. TB is receiving time and effort support from Genentech for a melanoma prevention study and is the principal investigator for melanoma clinical studies sponsored by Genentech, Amgen, and Replimune. JC serves as consultant/speaker for Amgen, BMS, Merck, Novartis, Pfizer, Roche. SGH serves as an advisor to Incyte. BC has received honoraria from Merck (DSMB member), Clinigen (Ad board), Nektar (Ad Board), research support from Galectin Therapeutics, Clinigen. BMS (to Institution) and AstraZeneca (to Institution). MOB discloses roles on Advisory boards (Merck, BMS, Novartis, EMD Serono, Sanofi, Pfizer, Immunocore, GSK); safety Review Committees (GSK, Adaptimmune), and research funding from Merck and Takara Bio. JL has received research funding to his institution from BMS, Immunocore, Polynoma, lovance and has participated in advisory boards from Regeneron and Array. DM directly is on speaker's bureaus for Pfizer, Merck, Astellas, and SeaGen. TS serves on Advisory boards for Immunocore and Castle Biosciences. EH is on the speakers' bureaus for Amgen and Castle Biosciences. SK is on the speakers' bureau for Janssen, Pfizer, Roche/Genentech, AstraZeneca. RW is on a Speaker's bureau for AstraZeneca. ABe has served as an advisor to Cardinal Health and Castle Biosciences. ABa is a consultant/speaker for Regeneron, Castle Biosciences, and receives research funding to her institution from Polynoma, Castle Biosciences, Pellepharm. CM has received grants, ad board and speaker fees from Novartis, BMS, Merck and Pfizer. VG is an investor in Medocity. DB: Research support- Natera; served on advisory boards for Merck, AstraZeneca, Novartis, Eli Lilly, Janssen, Oncocyte; speaker's bureau-Janssen, Guardant. JJP is a paid consultant for Polynoma.

Patient consent for publication Not applicable. 
Ethics approval The study was approved by Ethics Boards at each institution (IRB\#), as follows: University of Virginia Hospital (16223); Anschutz Cancer Pavilion, UC Denver (1134601); Huntsman Cancer Institute/University of Utah Health Care (55911); University of lowa Hospitals and Clinics (1133782); Mayo Clinic Cancer Center/Mayo Clinic Rochester (12-002308); The Angeles Clinic and Research Institute (1196134); Washington University School of Medicine (201205056); Intermountain Medical Center (1024288); University of Louisville (15.0039); CHU de Quebec, L'Hotel Dieu de Quebec (MP-20-2015-2318); Inova Melanoma and Skin Cancer Center (1152774); Mount Sinai Medical Center (12-16-H-03); Oregon Health and Science University (IRB00011848); Cancer Solutions (1197259). Participants all provided written informed consent for participation in this clinical trial.

Provenance and peer review Not commissioned; externally peer reviewed.

Data availability statement Data are available upon reasonable request.

Supplemental material This content has been supplied by the author(s). It has not been vetted by BMJ Publishing Group Limited (BMJ) and may not have been peer-reviewed. Any opinions or recommendations discussed are solely those of the author(s) and are not endorsed by BMJ. BMJ disclaims all liability and responsibility arising from any reliance placed on the content. Where the content includes any translated material, BMJ does not warrant the accuracy and reliability of the translations (including but not limited to local regulations, clinical guidelines, terminology, drug names and drug dosages), and is not responsible for any error and/or omissions arising from translation and adaptation or otherwise.

Open access This is an open access article distributed in accordance with the Creative Commons Attribution Non Commercial (CC BY-NC 4.0) license, which permits others to distribute, remix, adapt, build upon this work non-commercially, and license their derivative works on different terms, provided the original work is properly cited, appropriate credit is given, any changes made indicated, and the use is non-commercial. See http://creativecommons.org/licenses/by-nc/4.0/.

\section{ORCID iDs}

Craig L Slingluff http://orcid.org/0000-0002-6664-4373

Brendan Curti http://orcid.org/0000-0003-3948-2708

Jose Lutzky http://orcid.org/0000-0002-9503-2130

Suthee Rapisuwon http://orcid.org/0000-0002-1389-925X

\section{REFERENCES}

1 Kirkwood JM, Manola J, Ibrahim J, et al. A pooled analysis of eastern cooperative oncology group and intergroup trials of adjuvant highdose interferon for melanoma. Clin Cancer Res 2004;10:1670-7.

2 Swetter SM, Thompson JA, Albertini MR. Melanoma: cutaneous, version 2.2021. NCCN clinical practice guidelines in oncology: national comprehensive cancer network. Network NCC, 2021.

3 Reynolds SR, Oratz R, Shapiro RL, et al. Stimulation of CD8+ T cell responses to MAGE-3 and Melan A/MART-1 by immunization to a polyvalent melanoma vaccine. Int J Cancer 1997;72:972-6.

4 Reynolds SR, Celis E, Sette A, et al. HLA-independent heterogeneity of CD8+ T cell responses to MAGE-3, Melan-A/MART-1, gp100, tyrosinase, MC1R, and TRP-2 in vaccine-treated melanoma patients. $J$ Immunol 1998;161:6970-6.

5 Miller K, Abeles G, Oratz R, et al. Improved survival of patients with melanoma with an antibody response to immunization to a polyvalent melanoma vaccine. Cancer 1995;75:495-502.

6 Reynolds SR, Celis E, Sette A, et al. Identification of HLA-A*03, $A^{*} 11$ and $B^{*} 07-$ restricted melanoma-associated peptides that are immunogenic in vivo by vaccine-induced immune response (VIIR) analysis. J Immunol Methods 2000;244:59-67.

7 Bystryn JC, Zeleniuch-Jacquotte A, Oratz R, et al. Double-blind trial of a polyvalent, shed-antigen, melanoma vaccine. Clin Cancer Res 2001;7:1882-7.

8 Faries MB, Thompson JF, Cochran AJ, et al. Completion dissection or observation for sentinel-node metastasis in melanoma. $N$ Engl J Med 2017;376:2211-22.

9 Gabellini C, Trisciuoglio D, Desideri M, et al. Functional activity of CXCL8 receptors, CXCR1 and CXCR2, on human malignant melanoma progression. Eur J Cancer 2009;45:2618-27.

10 Korch C, Hall EM, Dirks WG, et al. Authentication of M14 melanoma cell line proves misidentification of MDA-MB-435 breast cancer cell line. Int J Cancer 2018;142:561-72.

11 Carey TE, Takahashi T, Resnick LA, et al. Cell surface antigens of human malignant melanoma: mixed hemadsorption assays for humoral immunity to cultured autologous melanoma cells. Proc Natl Acad Sci U S A 1976;73:3278-82.
12 Xing F, Persaud Y, Pratilas CA, et al. Concurrent loss of the PTEN and RB1 tumor suppressors attenuates RAF dependence in melanomas harboring (V600E)BRAF. Oncogene 2012;31:446-57.

13 Fogh J, Fogh JM, Orfeo T. One hundred and twenty-seven cultured human tumor cell lines producing tumors in nude mice. J Natl Cancer Inst 1977;59:221-6.

14 Adler A, Oraz R, Bystryn JC. In vitro cell-mediated immune responses induced by a polyvalent allogeneic melanoma vaccine. Cancer Biother 1995;10:211-24.

15 Hoos A, Eggermont AMM, Janetzki S, et al. Improved endpoints for cancer immunotherapy trials. J Natl Cancer Inst 2010;102:1388-97.

16 Grossmann KF, Othus M, Patel SP, et al. Final analysis of overall survival (OS) and relapse-free-survival (RFS) in the intergroup S1404 phase III randomized trial comparing either high-dose interferon (HDI) or ipilimumab to pembrolizumab in patients with high-risk resected melanoma. J Clin Oncol 2021;39:9501-01.

17 Schwartzentruber DJ, Lawson DH, Richards JM, et al. Gp100 peptide vaccine and interleukin-2 in patients with advanced melanoma. N Engl J Med 2011;364:2119-27.

18 Slingluff CL, Petroni GR, Olson W, et al. Helper T-cell responses and clinical activity of a melanoma vaccine with multiple peptides from MAGE and melanocytic differentiation antigens. J Clin Oncol 2008;26:4973-80.

19 Hunder NN, Wallen $\mathrm{H}$, Cao J, et al. Treatment of metastatic melanoma with autologous CD4+ T cells against NY-ESO-1. N Engl J Med 2008;358:2698-703.

20 Robbins PF, Kassim SH, Tran TLN, et al. A pilot trial using lymphocytes genetically engineered with an NY-ESO-1-reactive T-cell receptor: long-term follow-up and correlates with response. Clin Cancer Res 2015;21:1019-27.

21 Jones D, Pereira ER, Padera TP. Growth and immune evasion of lymph node metastasis. Front Oncol 2018;8:36.

22 Pawelec G. Immunosenescence and cancer. Biogerontology 2017;18:717-21.

23 Ramirez AG, Wages NA, Hu Y, et al. Defining the effects of age and gender on immune response and outcomes to melanoma vaccination: a retrospective analysis of a single-institution clinical trials' experience. Cancer Immunol Immunother 2015;64:1531-9.

24 Kugel CH, Douglass SM, Webster MR, et al. Age correlates with response to anti-PD1, reflecting age-related differences in intratumoral effector and regulatory T-cell populations. Clin Cancer Res 2018;24:5347-56.

25 Eggermont AMM, Suciu S, Testori A, et al. Ulceration and stage are predictive of interferon efficacy in melanoma: results of the phase III adjuvant trials EORTC 18952 and EORTC 18991. Eur J Cancer 2012;48:218-25.

26 Baurain J, Stas M, Hammouch F, et al. Association of primary melanoma ulceration and clinical benefit of adjuvant vaccination with tumor-specific antigenic peptides. J Clin Oncol 2009;27:3022-22.

27 Faries MB, Mozzillo N, Kashani-Sabet M, et al. Long-Term survival after complete surgical resection and adjuvant immunotherapy for distant melanoma metastases. Ann Surg Oncol 2017;24:3991-4000.

28 Morton DL, Mozzillo N, Thompson JF, et al. An international, randomized, phase III trial of Bacillus Calmette-Guerin (BCG) plus allogeneic melanoma vaccine (MCV) or placebo after complete resection of melanoma metastatic to regional or distant sites. J Clin Oncol 2007;25:8508.

29 Testori A, Richards J, Whitman E, et al. Phase III comparison of vitespen, an autologous tumor-derived heat shock protein gp96 peptide complex vaccine, with physician's choice of treatment for stage IV melanoma: the C-100-21 Study Group. J Clin Oncol 2008;26:955-62.

30 Cheever MA, Higano CS. Provenge (Sipuleucel-T) in prostate cancer: the first FDA-approved therapeutic cancer vaccine. Clin Cancer Res 2011;17:3520-6.

31 Schwartzentruber DJ, Lawson D, Richards J, et al. A phase III multi-institutional randomized study of immunization with the gp100: 209-217(210M) peptide followed by high-dose IL-2 compared with high-dose IL-2 alone in patients with metastatic melanoma. JCO 2009;27:CRA9011.

32 Merck announces phase 3 KEYNOTE-716 trial of anti-PD-1 therapy Keytruda to treat stage II melanoma meets primary endpoints. Available: Pharmbiz.com

33 Chen C, Wu B, Zhang C, et al. Immune-related adverse events associated with immune checkpoint inhibitors: an updated comprehensive disproportionality analysis of the FDA adverse event reporting system. Int Immunopharmacol 2021;95:107498.

34 Arora P, Talamo L, Dillon P, et al. Severe combined cardiac and neuromuscular toxicity from immune checkpoint blockade: an institutional case series. Cardiooncology 2020;6:21. 
35 Zhang R, Cai X-L, Liu L, et al. Type 1 diabetes induced by immune checkpoint inhibitors. Chin Med J 2020;133:2595-8.

36 Chang L-S, Barroso-Sousa R, Tolaney SM, et al. Endocrine toxicity of cancer immunotherapy targeting immune checkpoints. Endocr Rev 2019;40:17-65.
37 Long GV, Hauschild A, Santinami M, et al. Adjuvant dabrafenib plus trametinib in stage III BRAF-mutated melanoma. N Engl J Med 2017;377:1813-23. 